\title{
A longitudinal study of the diabetic skin and wound microbiome
}

\author{
Melissa Gardiner ${ }^{1}$, Mauro Vicaretti ${ }^{2,3}{ }^{\text {, Jill Sparks }}{ }^{4}$, Sunaina Bansal ${ }^{1}$, Stephen Bush ${ }^{5}$, Michael Liu ${ }^{1}$, \\ Aaron Darling ${ }^{1}$, Elizabeth Harry ${ }^{1}$, Catherine M Burke ${ }^{\text {Corresp. } 1}$ \\ ${ }^{1}$ The i3 institute, University of Technology Sydney, Sydney, New South Wales, Australia \\ 2 Medical School, University of Sydney, Sydney, New South Wales, Australia \\ 3 Westmead Hospital, Western Sydney Local Health District, Sydney, New South Wales, Australia \\ 4 Community Nursing, Western Sydney Local Health District, Sydney, New South Wales, Australia \\ 5 School of Mathematical and Physical Sciences, University of Technology Sydney, Sydney, New South Wales, Australia \\ Corresponding Author: Catherine M Burke \\ Email address: Catherine.Burke@uts.edu.au
}

Background. Type II diabetes is a chronic health condition which is associated with skin conditions including chronic foot ulcers and an increased incidence of skin infections. The skin microbiome is thought to play important roles in skin defence and immune functioning. Diabetes affects the skin environment, and this may perturb skin microbiome with possible implications for skin infections and wound healing. This study examines the skin and wound microbiome in type Il diabetes.

Methods. Eight type II diabetic subjects with chronic foot ulcers were followed over a time course of 10 weeks, sampling from both foot skin (swabs) and wounds (swabs and debrided tissue) every two weeks. A control group of 8 control subjects was also followed over 10 weeks, and skin swabs collected from the foot skin every two weeks. Samples were processed for DNA and subject to 16S rRNA gene PCR and sequencing of the $\mathrm{V} 4$ region.

Results. The diabetic skin microbiome was significantly less diverse than control skin. Community composition was also significantly different between diabetic and control skin, however the most abundant taxa were similar between groups, with differences driven by very low abundant members of the skin communities. Chronic wounds tended to be dominated by the most abundant skin Staphylococcus, while other abundant wound taxa differed by patient. No significant correlations were found between wound duration or healing status and the abundance of any particular taxa.

Discussion. The major difference observed in this study of the skin microbiome associated with diabetes was a significant reduction in diversity. The long-term effects of reduced diversity are not yet well understood, but are often associated with disease conditions. 


\section{Title: A longitudinal study of the diabetic skin and wound microbiome}

2 Melissa Gardiner ${ }^{1}$, Mauro Vicaretti²,3 ${ }^{2,}$ Jill Sparks $^{4}$, Sunaina Bansal ${ }^{1}$, Stephen Bush ${ }^{1}$, Michael

3 Liu $^{1}$, Aaron Darling ${ }^{1}$, Elizabeth Harry ${ }^{1}$, Catherine Burke ${ }^{1 *}$

4

5

6 1.The i3 institute, University of Technology Sydney, Sydney, NSW, Australia

7 2.Sydney Medical School, University of Sydney, Sydney, NSW, Australia

8 3.Westmead Hospital, Western Sydney Local Health District, Sydney, NSW, Australia

9 4.Community Nursing, Western Sydney Local Health District, Sydney, NSW, Australia

10

11 Corresponding author: Catherine Burke

12 Email address: Catherine.Burke@uts.edu.au 


\section{Abstract}

Background. Type II diabetes is a chronic health condition which is associated with skin conditions including chronic foot ulcers and an increased incidence of skin infections. The skin microbiome is thought to play important roles in skin defence and immune functioning. Diabetes affects the skin environment, and this may perturb skin microbiome with possible implications for skin infections and wound healing. This study examines the skin and wound microbiome in type II diabetes.

Methods. Eight type II diabetic subjects with chronic foot ulcers were followed over a time course of 10 weeks, sampling from both foot skin (swabs) and wounds (swabs and debrided tissue) every two weeks. A control group of eight subjects was also followed over 10 weeks, and skin swabs collected from the foot skin every two weeks. Samples were processed for DNA and subject to 16S rRNA gene PCR and sequencing of the V4 region.

Results. The diabetic skin microbiome was significantly less diverse than control skin. Community composition was also significantly different between diabetic and control skin, however the most abundant taxa were similar between groups, with differences driven by very low abundant members of the skin communities. Chronic wounds tended to be dominated by the most abundant skin Staphylococcus, while other abundant wound taxa differed by patient. No significant correlations were found between wound duration or healing status and the abundance of any particular taxa.

Discussion. The major difference observed in this study of the skin microbiome associated with diabetes was a significant reduction in diversity. The long-term effects of reduced diversity are not yet well understood, but are often associated with disease conditions. 


\section{Introduction}

40 Type II diabetes is one the fastest growing chronic diseases in the world today, predicted to rise

41 from 382 million people in 2013 to 592 million in 2035 (Guariguata et al. 2014). The disease is

42 characterised by persistently elevated blood glucose levels as a result of insufficient insulin

43 production or insulin resistance. This leads to many serious complications affecting the heart,

44 kidneys, eyes, blood vessels and nerves (World Health Organisation 2016). The development of

45 foot ulcers is the culmination of several of these complications, estimated to affect $15 \%$ of

46 diabetes sufferers (Reiber et al. 1995). These wounds are often slow to heal, difficult to treat,

47 and prone to infection. They have a severe impact on a patient's quality of life, and are estimated

48 to increase the risk of lower limb amputation by 15 fold (Australian Institute of Health and

49 Welfare 2008). The cost of treating these chronic wounds is estimated at up to $\$ 13$ billion

50 dollars annually in the US alone (Rice et al. 2014), and is set to rise with the increasing incidence

51 of diabetes worldwide.

52

53 Diabetes is associated with shifts in the gut microbiota (Karlsson et al. 2013; Qin et al. 2012), 54 and these shifts are thought to contribute to the onset of disease (Parekh et al. 2016; Zhang \& 55 Zhang 2013). Dysbiosis of the human microbiome is increasingly recognised to play a role in 56 many diseases, through mechanisms such as altered intestinal barrier function (Kelly et al. 2015), 57 triggering or exacerbating inflammation (Strober 2013) and regulation of energy metabolism 58 (Samuel et al. 2008). Given the physical changes that occur in the skin as a result of diabetes, 59 such as increased dryness and $\mathrm{pH}$, and glycosylation of structural skin proteins (Behm et al. 60 2012), it is feasible that diabetes may also affect the microbiome of the skin. 
62 As in the gut, the skin microbiome is thought to protect against infection via both competitive exclusion and direct inhibition (Bomar et al. 2016; Cogen et al. 2010b; Iwase et al. 2010; Shu et al. 2013), and have the potential to regulate skin immune function and wound healing (Kanno et al. 2011; Scales \& Huffnagle 2013). For example, the most common skin isolate, Staphylococcus epidermidis, has been shown to down-regulate inflammation following skin injury (Lai et al. 2009), and to up-regulate the production of antimicrobial peptides in the host (Lai et al. 2010), which work synergistically with antimicrobial peptides from S. epidermidis to inhibit pathogens such as Staphyloccocus aureus and Group A Streptococcus (Cogen et al. 2010a). Another skin commensal, Acinetobacter lwoffii, has been shown to protect against allergic sensitization and inflammation by promoting $\mathrm{T}_{\mathrm{H}} 1$ and anti-inflammatory responses in the skin (Fyhrquist et al. 2014). Given the importance of the skin microbiome in preventing infection, any shifts to these communities could affect their ability to protect against infection, and may have an effect on wound healing.

76 The aim of this study was to determine whether there are differences in the skin microbiome between persons with diabetes and healthy controls, and whether any members of the skin microbiome in diabetes are associated with those microbes that colonise chronic wounds during wound healing. We examined a cohort of eight diabetic and eight control individuals at six time points over a 10 -week period, by swabbing the skin on the soles of both feet, and collecting

81 swabs and debrided tissue from the chronic foot ulcers of the diabetic patients. The microbial

82 communities associated with these samples were assessed via high-throughput sequencing of the 83 V4 region of the bacterial 16S rRNA gene. 
Materials \& Methods

Study design, ethics approval, and sample collection

87 Ethical approval for the study was obtained from both the University of Technology Sydney Human Research Ethics Committee (approval number 2013000170), and the Western Sydney Local Health District Human Research Ethics Committee (approval number HREC2013/9/5.3(3809) AU RED LNR/13/WMEAD/294). Diabetic individuals and control subjects provided written consent for sample collection and all subsequent analyses.

92

Diabetic adults $(n=8)$ (Table 1) were selected for inclusion in the study based on medical diagnosis of type II diabetes, the presence of a chronic wound on one foot (chronic wound = present for six or more weeks) and no antibiotic therapy within the previous four weeks. Three swabs were collected for each diabetic subject every two weeks for a 10 week period using sterile rayon tipped swabs (Copan) that had been pre-moistened with a sterile solution of $0.15 \mathrm{M}$ $\mathrm{NaCl}$ and $0.1 \%$ Tween 20 . Two skin swabs were collected from intact foot skin 1 ) adjacent to the chronic wound (skin adjacent, SA) and 2) contralateral site to the chronic wound (skin contralateral, SC). Skin swabs were collected by firmly rubbing the moistened swab over the base of the foot skin surface for a period of 30 seconds. The whole base of the foot was used to maximise the DNA yield. Skin swab samples were taken prior to any cleaning of the skin surface that routinely took place before debridement of wound tissue. Chronic wounds were 104 cleaned by applying gauze soaked with Prontosan wound irrigation solution (B. Braun Medical, $\mathrm{UK}$ ) for ten 10 mins prior to sharp debridement of tissue from the top of the wound (wound 106 debridement, WD). Wound debridement samples were only taken where debridement was 
107 deemed to be necessary for the standard wound care. Wound swabs were taken after irrigation of

108 the wound with Prontosan to remove loose tissue, using a dry swab and the Z swab method

109 (wound swab, WS). The Z swab method was the routine method used in the clinic at the time of

110 sampling.

111 Control subjects $(n=8)$ (Table 1) were recruited from Sydney, Australia. The criteria for

112 inclusion were not to have been diagnosed as diabetic, between 50-80 years of age, and without

113 the use of antibiotics within the previous four weeks. Skin swabs were collected from the left and

114 right feet of control subjects as described above. Samples were taken from all participants every

115 two weeks for a 10-week period (6 time points in total). All samples were processed for DNA on

116 the day of collection, or stored at $4^{\circ} \mathrm{C}$ until processing the next day. These storage conditions

117 have been shown to adequately preserve the microbial profile of skin swab samples (Lauber et al. 118 2010).

119

Extraction of microbial DNA from skin and wound swabs and wound debridement tissue

121 Genomic DNA was extracted from all skin and wound samples using the BioStic DNA

122 extraction kit (MO BIO Laboratories, USA). Swab heads were cut off the plastic applicator using

123 sterile surgical scissors into the bead beating tube from the DNA extraction kit, before addition

124 of buffer CB1. For wound debridement tissue, the tissue was directly placed into the bead 125 beating tube. All subsequent steps were in accordance with the manufacturer's instructions, and 126 DNA was eluted in $50 \mu \mathrm{l}$ of solution CB5 (10mM Tris $\mathrm{pH} 8)$. The extracted DNA was 127 quantified on a Qubit ${ }^{\circledR} 2.0$ Fluorometer (Life Technologies, USA) with a Qubit ${ }^{\circledR}$ dsDNA HS 128 Assay Kit (Life Technologies, USA). 
131 A library of the V4 region of the 16S rRNA gene was prepared for Illumina sequencing from the

132 isolated microbial DNA samples. Samples were amplified using primers based on the Caporaso

133 et al design (Caporaso et al. 2012), which were modified to include eight nt rather than $12 \mathrm{nt}$

134 barcodes, and include a barcode on both the forward and reverse primer (V4_forward and

135 V4_reverse; Table 2). Different barcoded primers were used for each sample. For skin samples, 136 the V4 region was amplified from $500 \mathrm{pg}$ template DNA; for wound samples template DNA 137 started at $10 \mathrm{ng}$, but in some cases up to $50 \mathrm{ng}$ was used where a PCR product was not obtained 138 with lower amounts of template DNA. Each sample was subjected to 10 cycles of PCR with 0.5 $139 \mu \mathrm{M}$ each of V4_forward and V4_reverse barcoded primers in a $50 \mu \mathrm{l}$ PCR reaction that 140 contained $1 \times$ Taq core PCR buffer (Qiagen, Netherlands), $1 \times$ Q solution, $250 \mu \mathrm{M}$ dNTPs, and 141 1.25 U Taq DNA polymerase. Thermal cycling was carried out at $95^{\circ} \mathrm{C}$ for two minutes, 142 followed by 10 cycles of $95^{\circ} \mathrm{C}$ for 15 seconds, $50^{\circ} \mathrm{C}$ for 30 seconds and $72^{\circ} \mathrm{C}$ for 90 seconds, 143 followed by a final extension at $72^{\circ} \mathrm{C}$ for five minutes. Excess primer was removed via a 144 magnetic bead clean-up using 0.8 volume of Axygen ${ }^{\circledR}$ AxyPrep Mag beads (Corning, USA) and 145 the eluted amplicons were subjected to a further 20 cycles of PCR with $0.25 \mu \mathrm{M}$ enrichment 146 primers (Illumina_E_1 and Illumina_E_2; Table 2). The PCR reaction and cycling was 147 performed as described above, except that the annealing temperature was increased to $55^{\circ} \mathrm{C}$ and 14820 thermal cycles were performed. Following confirmation of the PCR product on a $1 \%$ agarose 149 gel, the amplicons were purified using Axygen ${ }^{\circledR}$ AxyPrep Mag beads (Corning, USA) and 150 quantified on a Qubit ${ }^{\circledR}$ 2.0 Fluorometer (Life Technologies, USA) with a Qubit ${ }^{\circledR}$ dsDNA HS 151 Assay Kit (Life Technologies, USA). Equimolar (2 ng) amounts of the 16S amplicons obtained 
152 for each skin and wound sample were then pooled and the molarity of the pooled amplicons

153 determined using a Bioanalyser High Sensitivity DNA chip (Agilent Technologies, USA).

154 Illumina sequencing and data analysis

155 The PCR amplicons from 264 samples (including positive and negative controls) were sequenced 156 over two separate runs on an Illumina Miseq using 500 cycle V2 kits. Sequences were 157 demultiplexed using phylosift (Darling et al. 2014) and read pairs merged using FLASH (Magoc 158 \& Salzberg 2011). Sequences were quality filtered and processed into OTUs using USEARCH v 159 1.8.1 (Edgar 2010) (fastq_filter command with the fastq_maxee option set to '2' to remove all 160 sequences with two or more expected errors). Further quality filtering and operational taxonomic 161 unit (OTU) clustering was carried out in QIIME (Caporaso et al. 2010b) version 1.9.0. The 162 split_libraries.py command was used with the -1 and -L options set to 240 and 260 respectively, 163 to remove sequences shorter than 240 and longer than 260 base pairs. Sequences were clustered 164 into OTUs at 97\% similarity using the pick_open_reference_otus.py script using default settings 165 except that singleton OTUs were removed, and the usearch61 method was used for chimera 166 filtering.

167 Taxonomy was assigned to OTUs (assign_taxonomy.py) using the UCLUST method (Edgar 168 2010) against the Greengenes (DeSantis et al. 2006) database pre-clustered at 97\% similarity, 169 accessed from the QIIME website 170 (ftp://greengenes.microbio.me/greengenes_release/gg_13_5/gg_13_8_otus.tar.gz).

171 Representative sequences from each OTU were aligned against the Greengenes alignment using 172 Pynast (Caporaso et al. 2010a) (align_seqs.py), OTUs which failed alignment were filtered from 173 the final OTU table (filter_otus_from_otu_table.py). A phylogenetic tree was built from aligned 174 representative OTU sequences (make_phylogeny.py script) using Fasttree2 (Price et al. 2010), 
175 with the $-\mathrm{r}$ option set to midpoint for tree rooting. For comparison, quality filtered sequences

176 were also clustered into OTUs using the UPARSE algorithm (Edgar 2013), with all downstream

177 analyses as per the QIIME workflow.

178 Diabetic skin samples adjacent to wounds were found to be more similar to wound than 179 contralateral skin samples (see Figure S1), and were removed so as not to confound comparisons 180 between diabetic and non-diabetic skin. To ensure more even sample sizes between the diabetic 181 and non-diabetic groups, only the right foot samples were included from the non-diabetic group 182 for all downstream analyses. Alpha diversity was calculated using Phyloseq (McMurdie \& 183 Holmes 2013) for the observed number of OTUs, Chao 1 and Shannon diversity indices on data 184 rarefied to 30000 sequences per sample. Significance testing was carried out on alpha diversity 185 estimates using the Wilcoxon rank sum test in R. per sample) using the weighted unifrac metric, and the generate_boxplots.py script used to compare unifrac distances between groups of samples. Futher beta diversity analyses, were carried out in Phyloseq, using weighted unifrac distances calculated from an OTU table with raw counts subject to variance stabilising transformation implemented in DEseq2 (Love et al. 2014) as described here (McMurdie \& Holmes 2014). Weighted unifrac distances matrices were also subject to principal coordinates analysis using the Phyloseq package, and significant differences in variance between groups (diabetic and control skin) were determined with PERMANOVA

194 (adonis function) implemented in the Vegan package (Oksanen et al. 2015) in R, using a nested 195 model formula (health/subject + subject) and the weighted unifrac distance matrix. 
196 The Wald test for differential abundance was used as implemented in the DESeq2 package in R.

197 Multivariate correlation analysis was carried out against OTUs and wound duration and area

198 using Pearson scores with Bonferroni correction, and p-values were determined via

199 bootstrapping with 100 permutations (implemented in QIIME using the

200 observation_metatdata_correlation.py command). OTU tables were filtered to remove OTUs

201 present in less than $10 \%$ of samples for both differential abundance and correlation tests.

202 A Random forest learning algorithm implemented in R (Liaw \& Wiener 2002) was used to 203 determine if diabetic status could be predicted from the foot skin microbiome. Skin samples

204 were randomly divided into two equal subsets (restricting samples from the same participant to

205 the same subset) for training and testing of learning algorithms. The variance stabilizing

206 transformed OTU table was filtered to include skin samples only, and to remove OTUs observed

207 in less than $10 \%$ of samples, and used as the input matrix for the Random forest algorithm. The

208 Random forest fitted on the training subset was created using bootstrapping of one third of the

209 training samples with replacement. As a general practice the rest of the samples were used as a

210 validation set in order to decrease the risk of over-fitting associated with classification

211 algorithms. An optimisation to minimise the out of bag error (classification error on validation

212 data) was used to obtain the optimal number of taxonomic units accessed at each iteration of

213 decision tree creation. Two hundred decision trees consisting of 30 OTUs evaluated at each node

214 of the tree were created. The Random forest model was then used to predict the health status of 215 the subjects in the test subset.

216

217 Analysis of the stability of skin microbial communities over time was carried out by comparing 218 intrapersonal weighted unifrac distances between the diabetic and control skin samples, along 
219 with intrapersonal distances for all samples. Kruskal-Wallis tests were used to determine 220 significant differences between groups.

221

222 Pearson's Product Moment Correlation was used to test for correlations between wound size or 223 duration and OTU abundance in wound samples as implemented in QIIME 224 (observation_metadata_correlation.py). P-values were calculated using bootstrapping with 100 225 permutations, and Bonferroni correction for multiple testing. Kruskal-Wallis tests for OTUs that 226 were differentially abundant in healing vs non-healing wounds were implemented in QIIME 227 (group_significance.py). Wounds were classified as healing or non-healing based on a reduction 228 in wound area since the last sampling time (healing) or no change or greater wound size area 229 since the last sampling (non-healing). OTU tables were filtered to remove OTUs present in less 230 than $10 \%$ of samples prior to testing.

231

232 Inter-visit weighted unifrac distances were compared to the overall degree of healing (1 - (final 233 wound area /initial wound area)) using the $1 \mathrm{~m}$ function of the stats package in R.

Quality filtered sequence data has been deposited in the European Nucleotide Archive under 236 study accession number PRJEB17696. A script containing the code used to process the data in R 237 is provided as supplementary data, along with all the necessary input files, including OTU table 238 and phylogenetic tree.

\section{Results}


241 The diabetic cohort $(n=8)$ consisted of 5 males and 3 females, with an average age of $68.9 \pm 8.2$ 242 (range $58-81$ ), average BMI of 35.4 \pm 5.9 (range $27.2-47.1$ ), and all had at least one foot ulcer

243 which had been present for a average time of 9.1 18.4 months (range 1.5-24 months). All

244 wounds were neuropathic, with the exception of Patient 6 where the wound was ischemic. Two

245 of the eight wounds healed during the course of sampling. Wounds were dressed with either

246 Allevyn foam (Smith and Nephew) to promote moist wound healing, Zetuvit dressing

247 (Hartmann) to remove excess wound exudate, Inadine antimicrobial dressing (10\% povidone-

248 iodine)(Johnson and Johnson), or Acticoat flex (antimicrobial silver coated) (Smith and

249 Nephew), as deemed appropriate by the treating podiatrist or wound care nurse. All wounds

250 were located on the plantar aspect of the foot. Details of the specific location of each wound,

251 along with size and treatment over time and are provided in Table S3.

252 The control cohort $(n=8)$ consisted of 2 males and 6 females, with an average age of $62.8 \pm 13.4$

253 (range 50-81), average BMI of 28.0 6.6 (range 20.4 - 37.9), and did not have wounds present on

254 the feet.

255 Sample processing, 16S PCR and sequencing

256 A total of 242 samples were collected from the diabetic and control cohorts, including 170 skin

257 swabs (85 diabetic and 85 control), 40 wound swabs and 32 wound debridement samples. Full

258 details for samples collected at each time point for each participant can be found in 259 supplementary Tables S1 (diabetic participants) and S2 (control participants).

260 DNA yields obtained from diabetic skin swabs varied from 0.51 to $600 \mathrm{ng}$, with a median of 8.5

261 ng. Three skin samples did not yield enough DNA to be measured by the Qubit assay, however

262 16S rRNA gene PCR products were still obtained. Control skin sample DNA yields ranged from 
2630.5 to $41.7 \mathrm{ng}$ (median 5.55), with 20 samples falling below the detection limit of the Qubit assay

$264(<5 \mathrm{pg} / \mu \mathrm{l})$. Of these 20 samples, PCR products were obtained for all but 3 . DNA yields from

265 wound swab samples ranged from $15 \mathrm{ng}$ to $5.6 \mu \mathrm{g}$ (median $760 \mathrm{ng}$ ) and for wound debridement

266 samples ranged from $170 \mathrm{ng}$ to $5.8 \mu \mathrm{g}$ (median $1.2 \mu \mathrm{g}$ ). One wound swab sample did not yield

267 enough DNA to be detected. Negative control swabs $(n=4)$ did not yield enough DNA to be

268 detected, and also did not yield detectable PCR products.

269 PCR products from the V4 region of the 16S rRNA gene were obtained for 257 of the 273

270 samples collected. Repeated attempts were made with increased amounts of template for those

271 samples that did not initially yield a PCR product, however no PCR product was obtained

272 (detailed in Tables S1 and S2). Amplicons from the remaining 257 samples were pooled and

273 paired-end sequenced over two separate MiSeq runs with V2-500 cycle kits. Sample from four

274 diabetic and four control subjects were sequenced in each run (Table S4). A median coverage of

27573599 sequences per sample was obtained (minimum 1683, maximum 297817). Negative

276 controls (two blank swab and 2 no DNA PCR controls) had between 1508 and 27840 sequences

277 assigned. The final sequencing coverage obtained for each sample can be found in Table S4.

278 Because negative control samples contained taxa that are similar to those found on skin (e.g.

279 Staphylococcus, Corynebacterium and Acinetobacter) specific taxa were not removed from the

280 data, rather samples with less than 30000 sequences $(n=5)$ were removed from the analysis,

281 based on the highest level of sequencing reads obtained from negative controls. A

282 PERMANOVA test was run on a weighted unfrac distance matrix generated from variance

283 stabilising transformed counts to assess the amount of variance attributable to the two different

284 sequencing runs, (run + subject). Sequencing run was a significant factor accounting for $3.0 \%$ of

285 the variance $(\mathrm{p}<0.001)$, while inter-individual differences accounted for $34.5 \%(\mathrm{p}<0.001)$. 
286

287 Diversity in all three groups was significantly different for observed richness, Chaol and 288 Shannon diversity indices (likelihood ratio test, $\mathrm{p}<0.01$ ). Diabetic skin was significantly less 289 diverse than control skin for richness and Chaol indices (Wilcoxon rank sum test, $\mathrm{p}<0.01$ ) 290 (Figure 1). Control skin had a median of 998.5 observed OTUs, compared to 435 for diabetic 291 skin. Wounds were also significantly less diverse than diabetic skin with a median of 145 292 observed OTUs.

The microbiome of diabetic skin is less diverse than control skin

The skin microbiome is significantly different between diabetic and control subjects

Skin microbial communities overall were significantly different between diabetic and control skin (Figure 2). A clear distinction can be observed between the sample types, and this was confirmed by a PERMANOVA test ( health/subject), where health (diabetes vs control) was a significant factor accounting for $11.7 \%$ of the variance $\left(\mathrm{R}^{2}=0.117, \mathrm{p}=0.001\right)$. Subject (interindividual differences) was the most significant factor accounting for $34.6 \%$ of the observed variance $\left(\mathrm{R}^{2}=0.346, \mathrm{p}=0.001\right)$.

Abundant taxa from skin are similar between persons with diabetes and healthy controls.

Despite the clear distinction between diabetic and control skin in the PCoA plot above, the most abundant taxa from both groups were similar. Foot skin communities from diabetic skin were dominated by the genera Staphylococcus, followed by Acinetobacter and Corynebacterium, then unclassified Enterobacteriacea. Control skin was dominated by the genera Staphylococcus, followed by Acinetobacter, Kocuria, Corynebacterium and Micrococcus,(Figure 3). 
306 To determine which OTUs were contributing to the significant difference detected in the

307 PERMANOVA analysis, the Wald test as implemented in the DESeq2 package (Love et al.

308 2014), was carried out. Sixty-nine OTUs were identified as significantly different in abundance

309 (adjusted $\mathrm{p}<0.05$ ), all with an average abundance of less than 1\%. A full list of the results can

310 be found in Table S5. Similar results were found when re-running the analysis at the Genera

311 level, with 24 genera identified as significantly different, but all at an average relative abundance

312 of less then $1 \%$ (Table S6).

313 The foot skin microbiome may predict diabetic status

314 Despite only low abundance OTUs showing significant differences between diabetic and non315 diabetic skin, a Random Forrest classifier was able to predict diabetic status from the foot skin 316 microbiome. The model achieved an overall accuracy of $85.0 \%$, with a sensitivity of $79.2 \%$, and 317 specificity of $93.8 \%$. The negative predictive value $(75.0 \%)$ was lower than the positive 318 predictive value (95.0\%). The classifier's Gini index provided a list of 106 OTUs that were 319 important in the classification task (Table S7); the majority were low abundance OTUs (103 320 OTUs $<1 \%$ average relative abundance), and the majority of these were more abundant in 321 control than diabetic skin (75 OTUs).

Stability of the diabetic skin microbiome over time

325 Longitudinal analysis of the skin microbiome over time showed a trend of lower stability for 326 diabetic skin than non-diabetic skin (Figure 4), however this difference did not reach significance 327 ( $\mathrm{p}=0.09)$, while both control and diabetic skin intrapersonal differences over time were 328 significantly smaller (i.e. more stable) than inter-individual differences $(\mathrm{p}<0.05)$. 
330 Microbiota of chronic diabetic wounds overlap with skin and differ between patient

331 Wound swab and debridement samples were similar in taxonomic composition, and the top ten

332 OTUs from all wounds per patient are shown in Figure 5. The most abundant OTU detected in 333 wounds was also the most abundant OTU found on skin, Staphylococcus sp. (OTU 1084865), 334 and was present in the wounds of all eight patients. Other skin associated OTUs found in 335 wounds included Corynebacterium (OTU 1011712), which was in the top 10 OTUs in six out of 336 eight patient's wounds.

337

The Wald test for differential abundance between diabetic skin and wounds identified four OTUs that were significantly more abundant across all wounds (two classified as Enterobactericaeae, one as Serratia and one as Finegoldia). The complete list of results can be found in Table S8.

342 The top 10 OTUs in wounds per patient over time are shown in Figure 6. Of the eight wounds, 343 six are dominated by the most abundant skin OTU, at the majority of time points measured 344 (Staphylococcus OTU 1084865). Only Patients 6 and 10 showed wound profiles dominated by non-skin associated taxa across the time period surveyed. No significant correlations were found 346 between any abundant OTUs (average abundance $>1 \%$ ) and wound duration or healing status. 347 No significant correlation was found between the overall degree of wound healing, and inter-visit 348 weighted unifrac distances in individual wounds (Figure S2, p=0.29). However, some 349 interesting observations were made that correlated to clinical events. For example, the wound of 350 Patient 6 had been present for 24 months at the start of the study. It was dominated by 351 Enterobacteriacaea and showed little healing until time point 3, which coincided with an 
352 angioplasty procedure to improve blood flow to the foot. This was followed by resolution of the

353 wound within two weeks. When Patient 7 presented to the clinic, the wound had been present

354 for 12 months, and was dominated by an OTU from the Neisseriaceae family. Following the

355 standard treatment of debridement and wound dressing, rapid healing was observed, as well as a

356 shift to a community dominated by the most abundant skin OTU.

357

358 Discussion

359 This study aimed to compare the skin microbiome between persons with diabetes and healthy 360 control individuals over time. We additionally sought to characterise the wound microbiota in 361 diabetic foot ulcers over time and determine if any members of the skin microbiome were 362 correlated to the wound microbiome or wound healing.

363 The microbiome from diabetic skin was significantly different to that of control skin, however 364 this difference was not driven by the most abundant members of the skin community. The top 10 365 most abundant OTUs per person were similar in abundance and not significantly different 366 between groups. Many low abundance OTUs were identified as significantly different, with the 367 vast majority of these being more abundant in control skin. One limitation of this study is that, 368 although commonly used in microbiome studies (Cope et al. 2017; David et al. 2014; Halfvarson 369 et al. 2017; Smith et al. 2016), the V4 region of the $16 \mathrm{~S}$ rRNA gene does not allow 370 differentiation between Staphylococcus aureus and other Staphylococcus species found on skin, 371 such as Staphylococcus epidermidis (Conlan et al. 2012). Additionally, the V4 primers have 372 mismatches that prevent detection of Propionibacterium, an important genera in the skin 373 microbiome (Kuczynski et al. 2011). The clinical consequences of these organisms may be 
374 important, and this should be taken into consideration for the experimental design of future 375 studies (Gohl et al. 2016; Meisel et al. 2016).

376 We observed a significant reduction in alpha diversity and a trend of decreased stability (non377 significant) of diabetic skin microbiomes compared to non-diabetic skin. This is in contrast to a 378 previous study of diabetic skin (Redel et al. 2013) where the opposite result was observed. It is 379 possible that changes to the skin environment associated with diabetes, such as increased $\mathrm{pH}$ 380 (Yosipovitch et al. 1993) advanced glycation end products in the skin matrix (Gkogkolou \& 381 Bohm 2012), or increased levels of skin inflammation (Tellechea et al. 2013) could drive a 382 decrease in diversity. It is also possible that activities associated with diabetes, such as increased exposure to antibiotics (Mor et al. 2016), contribute to the observed effect despite our attempts to control for recent antibiotic exposure as a confounding variable. Another limitation of the current study is the small sample size, and as such this result should be confirmed on a larger 386 cohort.

If skin microbiome diversity is depleted in people with diabetes, what are the implications for the health of diabetic skin? While in some body sites an increase is microbial diversity is associated with disease states, particularly the vagina (van de Wijgert et al. 2014), decreased diversity of the microbiome has frequently been correlated with disease and inflammation in the skin (Alekseyenko et al. 2013; Ellebrecht et al. 2016; Seite et al. 2014; Williams \& Gallo 2015), gut (Giloteaux et al. 2016; Sze \& Schloss 2016) and airways (Yu et al. 2015). However it is not known whether decreased diversity in these sites is a cause or merely an indicator of inflammation. Diversity is commonly used as an indicator of ecosystem health, with decreased diversity typically signalling a disturbed and less resilient state (Oliver et al. 2015). In the 
397 overgrow, and these may be capable of triggering inflammation and triggering or exacerbating a

398 disease state. Alternatively, inflammation could be triggered by genetic and environmental

399 factors, and the inflammation itself could drive down bacterial diversity by creating an 400 inhospitable growth environment.

401 Patients with diabetes enrolled in this study had no exposure to antibiotics within the previous 4 402 weeks, so as not to confound the comparison between diabetic and control skin. This meant that 403 the foot ulcers analysed in this study were considered to be clinically non-infected wounds. No 404 significant correlations were found between any OTU in diabetic skin or wounds with wound 405 size, duration or healing status. This is possibly due to the small sample size, as a previous study 406 found correlations between the relative abundance of specific bacterial taxa and ulcer duration 407 and depth (e.g. Staphylococcus was negatively correlated with wound duration) (Gardner et al. 408 2013). Another possible limitation of this study is the use of the z-swab method which samples 409 across the entire wound base regardless of size, as this will possibly increase heterogeneity with 410 increasing wound size.

411 A recent longitudinal study of wounds found a negative correlation between wound microbiota 412 stability and time to heal (Loesche et al. 2017). We did not find any such correlation here when 413 comparing degree of healing to between visit weighted unifrac distances (Figure S2), although 414 again our sample size was smaller, as was the length of time patients were followed.

415 The overall composition of the diabetic wound microbiota described here is in agreement with a 416 survey of 910 chronic diabetic foot ulcers, where a dominance of Staphylococcus, as well as 417 Pseudomonas, Corynebacterium, Streptococcus and Finegoldia (among others) was found 418 (Wolcott et al. 2016). Gardiner et al. (2013) found that diabetic ulcers clustered into three types, 
419 depending on the dominant taxa in the wounds, which were Staphylococcus, Streptococcus, or a

420 mixture of anaerobic bacteria or Proteobacteria. Similar results were found in a later study

421 where 2 wound clusters were dominated by either Staphylococcus or Streptococcus, and genera

422 such as Corynebacterium and Finegoldia were frequently observed (Loesche et al. 2017). These

423 same genera were observed in most wounds here, while other genera such as Serratia and

424 Proteus were specific to individuals.

425 Other studies of diabetic foot ulcers have reported contrasting results, such as a dominance of

426 Corynebacterium (Dowd et al. 2008), while a recent study found that Staphylococcus were

427 common in new ulcers, but not in recurring ulcers (Smith et al. 2016). One trend that was

428 consistent across several studies was that the microbial profile from diabetic ulcers was variable,

429 with no one typical diabetic ulcer microbiota apparent.

\section{Conclusions}

431 The major effect associated with diabetes observed here was a significant reduction in the

432 diversity of the skin microbiome. The cohort of this study was small, and these observations

433 should be verified in a larger study. The long-term effects of reduced diversity are not yet well

434 understood, but low diversity continues to be linked to disease and poor health outcomes (Hua et

435 al. 2016; Miller et al. 2016; Rook 2013). One possible effect is increased infection susceptibility

436 (Seto et al. 2014), and it is intriguing to consider whether decreased skin microbiome diversity

437 could be contributing to the high incidence of skin and wound infections associated with this

438 disease (Peleg et al. 2007). There are of course many other well-documented factors such as

439 immune dysfunction that can contribute to an increased rate of infections (Geerlings \& 
440 Hoepelman 1999), however the skin microbiome may be an as yet unconsidered contributor to

441 this phenomenon.

\section{Acknowledgements}

443 We would like to acknowledge Westmead Hospital and the Podiatry Clinic Staff who kindly

444 assisted in the collection of samples. We also acknowledge Professor Mark Morrison and Dr

445 Paraic O Cuiv for helpful discussions about the study design.

446

447 
448

449

450

451

452

453

454

455

456

457

458

459

460

461

462

463

464

465

466

467

468

469

470

471

472

473

474

475

476

477

478

479

480

481

482

483

484

485

486

487

488

489

490

491

492

493

\section{References}

Alekseyenko AV, Perez-Perez GI, De Souza A, Strober B, Gao Z, Bihan M, Li K, Methe BA, and Blaser MJ. 2013. Community differentiation of the cutaneous microbiota in psoriasis. Microbiome 1:31. 10.1186/2049-2618-1-31

Australian Institute of Health and Welfare. 2008. Diabetes: Australian Facts 2008. Canberra: AIHW.

Behm B, Schreml S, Landthaler M, and Babilas P. 2012. Skin signs in diabetes mellitus. J Eur Acad Dermatol Venereol 26:1203-1211. 10.1111/j.1468-3083.2012.04475.x

Bomar L, Brugger SD, Yost BH, Davies SS, and Lemon KP. 2016. Corynebacterium accolens Releases Antipneumococcal Free Fatty Acids from Human Nostril and Skin Surface Triacylglycerols. MBio 7:e01725-01715. 10.1128/mBio.01725-15

Caporaso JG, Bittinger K, Bushman FD, DeSantis TZ, Andersen GL, and Knight R. 2010a. PyNAST: a flexible tool for aligning sequences to a template alignment. Bioinformatics 26:266-267. 10.1093/bioinformatics/btp636

Caporaso JG, Kuczynski J, Stombaugh J, Bittinger K, Bushman FD, Costello EK, Fierer N, Pena AG, Goodrich JK, Gordon JI, Huttley GA, Kelley ST, Knights D, Koenig JE, Ley RE, Lozupone CA, McDonald D, Muegge BD, Pirrung M, Reeder J, Sevinsky JR, Turnbaugh PJ, Walters WA, Widmann J, Yatsunenko T, Zaneveld J, and Knight R. 2010b. QIIME allows analysis of high-throughput community sequencing data. Nat Methods 7:335-336. 10.1038/nmeth.f.303

Caporaso JG, Lauber CL, Walters WA, Berg-Lyons D, Huntley J, Fierer N, Owens SM, Betley J, Fraser L, Bauer M, Gormley N, Gilbert JA, Smith G, and Knight R. 2012. Ultra-highthroughput microbial community analysis on the Illumina HiSeq and MiSeq platforms. ISME J 6:1621-1624. 10.1038/ismej.2012.8

Cogen AL, Yamasaki K, Muto J, Sanchez KM, Crotty Alexander L, Tanios J, Lai Y, Kim JE, Nizet V, and Gallo RL. 2010a. Staphylococcus epidermidis antimicrobial delta-toxin (phenol-soluble modulin-gamma) cooperates with host antimicrobial peptides to kill group A Streptococcus. PLoS One 5:e8557. 10.1371/journal.pone.0008557

Cogen AL, Yamasaki K, Sanchez KM, Dorschner RA, Lai Y, MacLeod DT, Torpey JW, Otto M, Nizet V, Kim JE, and Gallo RL. 2010b. Selective antimicrobial action is provided by phenol-soluble modulins derived from Staphylococcus epidermidis, a normal resident of the skin. J Invest Dermatol 130:192-200. 10.1038/jid.2009.243

Conlan S, Kong HH, and Segre JA. 2012. Species-level analysis of DNA sequence data from the NIH Human Microbiome Project. PLoS One 7:e47075. 10.1371/journal.pone.0047075

Cope EK, Goldberg AN, Pletcher SD, and Lynch SV. 2017. Compositionally and functionally distinct sinus microbiota in chronic rhinosinusitis patients have immunological and clinically divergent consequences. Microbiome 5:53. 10.1186/s40168-017-0266-6

Darling AE, Jospin G, Lowe E, Matsen FAt, Bik HM, and Eisen JA. 2014. PhyloSift: phylogenetic analysis of genomes and metagenomes. PeerJ 2:e243. 10.7717/peerj.243

David LA, Maurice CF, Carmody RN, Gootenberg DB, Button JE, Wolfe BE, Ling AV, Devlin AS, Varma Y, Fischbach MA, Biddinger SB, Dutton RJ, and Turnbaugh PJ. 2014. Diet rapidly and reproducibly alters the human gut microbiome. Nature 505:559-563. 10.1038 /nature 12820

DeSantis TZ, Hugenholtz P, Larsen N, Rojas M, Brodie EL, Keller K, Huber T, Dalevi D, Hu P, and Andersen GL. 2006. Greengenes, a chimera-checked 16S rRNA gene database and 
workbench compatible with ARB. Appl Environ Microbiol 72:5069-5072. 10.1128/AEM.03006-05

Dowd SE, Wolcott RD, Sun Y, McKeehan T, Smith E, and Rhoads D. 2008. Polymicrobial nature of chronic diabetic foot ulcer biofilm infections determined using bacterial tag encoded FLX amplicon pyrosequencing (bTEFAP). PLoS One 3:e3326. 10.1371/journal.pone.0003326

Edgar RC. 2010. Search and clustering orders of magnitude faster than BLAST. Bioinformatics 26:2460-2461. 10.1093/bioinformatics/btq461

Edgar RC. 2013. UPARSE: highly accurate OTU sequences from microbial amplicon reads. Nat Methods 10:996-998. 10.1038/nmeth.2604

Ellebrecht CT, Srinivas G, Bieber K, Banczyk D, Kalies K, Kunzel S, Hammers CM, Baines JF, Zillikens D, Ludwig RJ, and Westermann J. 2016. Skin microbiota-associated inflammation precedes autoantibody induced tissue damage in experimental epidermolysis bullosa acquisita. J Autoimmun 68:14-22. 10.1016/j.jaut.2015.08.007

Fyhrquist N, Ruokolainen L, Suomalainen A, Lehtimaki S, Veckman V, Vendelin J, Karisola P, Lehto M, Savinko T, Jarva H, Kosunen TU, Corander J, Auvinen P, Paulin L, von Hertzen L, Laatikainen T, Makela M, Haahtela T, Greco D, Hanski I, and Alenius H. 2014. Acinetobacter species in the skin microbiota protect against allergic sensitization and inflammation. J Allergy Clin Immunol 134:1301-1309 e1311. 10.1016/j.jaci.2014.07.059

Gardner SE, Hillis SL, Heilmann K, Segre JA, and Grice EA. 2013. The neuropathic diabetic foot ulcer microbiome is associated with clinical factors. Diabetes 62:923-930. $10.2337 / \mathrm{db} 12-0771$

Geerlings SE, and Hoepelman AI. 1999. Immune dysfunction in patients with diabetes mellitus (DM). FEMS Immunol Med Microbiol 26:259-265.

Giloteaux L, Goodrich JK, Walters WA, Levine SM, Ley RE, and Hanson MR. 2016. Reduced diversity and altered composition of the gut microbiome in individuals with myalgic encephalomyelitis/chronic fatigue syndrome. Microbiome 4:30. 10.1186/s40168-0160171-4

Gkogkolou P, and Bohm M. 2012. Advanced glycation end products: Key players in skin aging? Dermatoendocrinol 4:259-270. 10.4161/derm.22028

Gohl DM, Vangay P, Garbe J, MacLean A, Hauge A, Becker A, Gould TJ, Clayton JB, Johnson TJ, Hunter R, Knights D, and Beckman KB. 2016. Systematic improvement of amplicon marker gene methods for increased accuracy in microbiome studies. Nat Biotechnol 34:942-949. 10.1038/nbt.3601

Guariguata L, Whiting DR, Hambleton I, Beagley J, Linnenkamp U, and Shaw JE. 2014. Global estimates of diabetes prevalence for 2013 and projections for 2035. Diabetes Res Clin Pract 103:137-149. 10.1016/j.diabres.2013.11.002

Halfvarson J, Brislawn CJ, Lamendella R, Vazquez-Baeza Y, Walters WA, Bramer LM, D'Amato M, Bonfiglio F, McDonald D, Gonzalez A, McClure EE, Dunklebarger MF, Knight R, and Jansson JK. 2017. Dynamics of the human gut microbiome in inflammatory bowel disease. Nat Microbiol 2:17004. 10.1038/nmicrobiol.2017.4

Hua X, Goedert JJ, Pu A, Yu G, and Shi J. 2016. Allergy associations with the adult fecal microbiota: Analysis of the American Gut Project. EBioMedicine 3:172-179. 10.1016/j.ebiom.2015.11.038 
539

540

541

542

543

544

545

546

547

548

549

550

551

552

553

554

555

556

557

558

559

560

561

562

563

564

565

566

567

568

569

570

571

572

573

574

575

576

577

578

579

580

581

582

583

584

Iwase T, Uehara Y, Shinji H, Tajima A, Seo H, Takada K, Agata T, and Mizunoe Y. 2010. Staphylococcus epidermidis Esp inhibits Staphylococcus aureus biofilm formation and nasal colonization. Nature 465:346-349. 10.1038/nature09074

Kanno E, Kawakami K, Ritsu M, Ishii K, Tanno H, Toriyabe S, Imai Y, Maruyama R, and Tachi M. 2011. Wound healing in skin promoted by inoculation with Pseudomonas aeruginosa PAO1: The critical role of tumor necrosis factor-alpha secreted from infiltrating neutrophils. Wound Repair Regen 19:608-621. 10.1111/j.1524-475X.2011.00721.x

Karlsson FH, Tremaroli V, Nookaew I, Bergstrom G, Behre CJ, Fagerberg B, Nielsen J, and Backhed F. 2013. Gut metagenome in European women with normal, impaired and diabetic glucose control. Nature 498:99-103. 10.1038/nature12198

Kelly CJ, Zheng L, Campbell EL, Saeedi B, Scholz CC, Bayless AJ, Wilson KE, Glover LE, Kominsky DJ, Magnuson A, Weir TL, Ehrentraut SF, Pickel C, Kuhn KA, Lanis JM, Nguyen V, Taylor CT, and Colgan SP. 2015. Crosstalk between Microbiota-Derived Short-Chain Fatty Acids and Intestinal Epithelial HIF Augments Tissue Barrier Function. Cell Host Microbe 17:662-671. 10.1016/j.chom.2015.03.005

Kuczynski J, Lauber CL, Walters WA, Parfrey LW, Clemente JC, Gevers D, and Knight R. 2011. Experimental and analytical tools for studying the human microbiome. Nat Rev Genet 13:47-58. 10.1038/nrg3129

Lai Y, Cogen AL, Radek KA, Park HJ, Macleod DT, Leichtle A, Ryan AF, Di Nardo A, and Gallo RL. 2010. Activation of TLR2 by a small molecule produced by Staphylococcus epidermidis increases antimicrobial defense against bacterial skin infections. J Invest Dermatol 130:2211-2221. 10.1038/jid.2010.123

Lai Y, Di Nardo A, Nakatsuji T, Leichtle A, Yang Y, Cogen AL, Wu ZR, Hooper LV, Schmidt RR, von Aulock S, Radek KA, Huang CM, Ryan AF, and Gallo RL. 2009. Commensal bacteria regulate Toll-like receptor 3-dependent inflammation after skin injury. Nat Med 15:1377-1382. 10.1038/nm.2062

Lauber CL, Zhou N, Gordon JI, Knight R, and Fierer N. 2010. Effect of storage conditions on the assessment of bacterial community structure in soil and human-associated samples. FEMS Microbiol Lett 307:80-86. 10.1111/j.1574-6968.2010.01965.x

Liaw A, and Wiener M. 2002. Classification and Regression by randomForest. $R$ News 2:18-22.

Loesche M, Gardner SE, Kalan L, Horwinski J, Zheng Q, Hodkinson BP, Tyldsley AS, Franciscus CL, Hillis SL, Mehta S, Margolis DJ, and Grice EA. 2017. Temporal Stability in Chronic Wound Microbiota Is Associated With Poor Healing. J Invest Dermatol 137:237-244. 10.1016/j.jid.2016.08.009

Love MI, Huber W, and Anders S. 2014. Moderated estimation of fold change and dispersion for RNA-seq data with DESeq2. Genome Biol 15:550. 10.1186/s13059-014-0550-8

Magoc T, and Salzberg SL. 2011. FLASH: fast length adjustment of short reads to improve genome assemblies. Bioinformatics 27:2957-2963. 10.1093/bioinformatics/btr507

McMurdie PJ, and Holmes S. 2013. phyloseq: an R package for reproducible interactive analysis and graphics of microbiome census data. PLoS One 8:e61217.

10.1371/journal.pone.0061217

McMurdie PJ, and Holmes S. 2014. Waste not, want not: why rarefying microbiome data is inadmissible. PLoS Comput Biol 10:e1003531. 10.1371/journal.pcbi.1003531

Meisel JS, Hannigan GD, Tyldsley AS, SanMiguel AJ, Hodkinson BP, Zheng Q, and Grice EA. 2016. Skin Microbiome Surveys Are Strongly Influenced by Experimental Design. $J$ Invest Dermatol 136:947-956. 10.1016/j.jid.2016.01.016 
585

586

587

588

589

590

591

592

593

594

595

596

597

598

599

600

601

602

603

604

605

606

607

608

609

610

611

612

613

614

615

616

617

618

619

620

621

622

623

624

625

626

627

628

629

Miller GE, Engen PA, Gillevet PM, Shaikh M, Sikaroodi M, Forsyth CB, Mutlu E, and Keshavarzian A. 2016. Lower Neighborhood Socioeconomic Status Associated with Reduced Diversity of the Colonic Microbiota in Healthy Adults. PLoS One 11:e0148952. 10.1371/journal.pone.0148952

Mor A, Berencsi K, Nielsen JS, Rungby J, Friborg S, Brandslund I, Christiansen JS, Vaag A, Beck-Nielsen H, Sorensen HT, and Thomsen RW. 2016. Rates of Community-based Antibiotic Prescriptions and Hospital-treated Infections in Individuals With and Without Type 2 Diabetes: A Danish Nationwide Cohort Study, 2004-2012. Clin Infect Dis. 10.1093/cid/ciw345

Oksanen J, Blanchet FG, Kindt R, Legendre P, Minchin PR, O'Hara RB, Simpson GL, Solymos P, Henry M, Stevens H, and Wagner H. 2015. vegan: Community Ecology Package.

Oliver TH, Heard MS, Isaac NJ, Roy DB, Procter D, Eigenbrod F, Freckleton R, Hector A, Orme CD, Petchey OL, Proenca V, Raffaelli D, Suttle KB, Mace GM, Martin-Lopez B, Woodcock BA, and Bullock JM. 2015. Biodiversity and Resilience of Ecosystem Functions. Trends Ecol Evol 30:673-684. 10.1016/j.tree.2015.08.009

Parekh PJ, Nayi VR, Johnson DA, and Vinik AI. 2016. The Role of Gut Microflora and the Cholinergic Anti-inflammatory Neuroendocrine System in Diabetes Mellitus. Front Endocrinol (Lausanne) 7:55. 10.3389/fendo.2016.00055

Peleg AY, Weerarathna T, McCarthy JS, and Davis TM. 2007. Common infections in diabetes: pathogenesis, management and relationship to glycaemic control. Diabetes Metab Res Rev 23:3-13. 10.1002/dmrr.682

Price MN, Dehal PS, and Arkin AP. 2010. FastTree 2--approximately maximum-likelihood trees for large alignments. PLoS One 5:e9490. 10.1371/journal.pone.0009490

Qin J, Li Y, Cai Z, Li S, Zhu J, Zhang F, Liang S, Zhang W, Guan Y, Shen D, Peng Y, Zhang D, Jie Z, Wu W, Qin Y, Xue W, Li J, Han L, Lu D, Wu P, Dai Y, Sun X, Li Z, Tang A, Zhong S, Li X, Chen W, Xu R, Wang M, Feng Q, Gong M, Yu J, Zhang Y, Zhang M, Hansen T, Sanchez G, Raes J, Falony G, Okuda S, Almeida M, LeChatelier E, Renault P, Pons N, Batto JM, Zhang Z, Chen H, Yang R, Zheng W, Li S, Yang H, Wang J, Ehrlich SD, Nielsen R, Pedersen O, Kristiansen K, and Wang J. 2012. A metagenome-wide association study of gut microbiota in type 2 diabetes. Nature 490:55-60. 10.1038/nature11450

Redel H, Gao Z, Li H, Alekseyenko AV, Zhou Y, Perez-Perez GI, Weinstock G, Sodergren E, and Blaser MJ. 2013. Quantitation and composition of cutaneous microbiota in diabetic and nondiabetic men. J Infect Dis 207:1105-1114. 10.1093/infdis/jit005

Reiber GE, Boyko EJ, and Smith DG. 1995. Lower Extremity Foot Ulcers and Amputations in Diabetes. In: NDDG, ed. Diabetes in America. 2nd edition ed. Bethsada: National Institutes of Health.

Rice JB, Desai U, Cummings AK, Birnbaum HG, Skornicki M, and Parsons NB. 2014. Burden of diabetic foot ulcers for medicare and private insurers. Diabetes Care 37:651-658. $10.2337 / \mathrm{dc} 13-2176$

Rook GA. 2013. Regulation of the immune system by biodiversity from the natural environment: an ecosystem service essential to health. Proc Natl Acad Sci US A 110:18360-18367. 10.1073/pnas. 1313731110

Samuel BS, Shaito A, Motoike T, Rey FE, Backhed F, Manchester JK, Hammer RE, Williams SC, Crowley J, Yanagisawa M, and Gordon JI. 2008. Effects of the gut microbiota on 
630

631

632

633

634

635

636

637

638

639

640

641

642

643

644

645

646

647

648

649

650

651

652

653

654

655

656

657

658

659

660

661

662

663

664

665

666

667

668

669

670

671

672

673

674

675 host adiposity are modulated by the short-chain fatty-acid binding $\mathrm{G}$ protein-coupled

receptor, Gpr41. Proc Natl Acad Sci U S A 105:16767-16772. 10.1073/pnas.0808567105

Scales BS, and Huffnagle GB. 2013. The microbiome in wound repair and tissue fibrosis. $J$ Pathol 229:323-331. 10.1002/path.4118

Seite S, Flores GE, Henley JB, Martin R, Zelenkova H, Aguilar L, and Fierer N. 2014. Microbiome of affected and unaffected skin of patients with atopic dermatitis before and after emollient treatment. J Drugs Dermatol 13:1365-1372.

Seto CT, Jeraldo P, Orenstein R, Chia N, and DiBaise JK. 2014. Prolonged use of a proton pump inhibitor reduces microbial diversity: implications for Clostridium difficile susceptibility. Microbiome 2:42. 10.1186/2049-2618-2-42

Shu M, Wang Y, Yu J, Kuo S, Coda A, Jiang Y, Gallo RL, and Huang CM. 2013. Fermentation of Propionibacterium acnes, a commensal bacterium in the human skin microbiome, as skin probiotics against methicillin-resistant Staphylococcus aureus. PLoS One 8:e55380. 10.1371/journal.pone.0055380

Smith K, Collier A, Townsend EM, O'Donnell LE, Bal AM, Butcher J, Mackay WG, Ramage G, and Williams C. 2016. One step closer to understanding the role of bacteria in diabetic foot ulcers: characterising the microbiome of ulcers. BMC Microbiol 16:54. 10.1186/s 12866-016-0665-Z

Strober W. 2013. Impact of the gut microbiome on mucosal inflammation. Trends Immunol 34:423-430. 10.1016/j.it.2013.07.001

Sze MA, and Schloss PD. 2016. Looking for a Signal in the Noise: Revisiting Obesity and the Microbiome. MBio 7. 10.1128/mBio.01018-16

Tellechea A, Kafanas A, Leal EC, Tecilazich F, Kuchibhotla S, Auster ME, Kontoes I, Paolino J, Carvalho E, Nabzdyk LP, and Veves A. 2013. Increased skin inflammation and blood vessel density in human and experimental diabetes. Int J Low Extrem Wounds 12:4-11. $10.1177 / 1534734612474303$

van de Wijgert JH, Borgdorff H, Verhelst R, Crucitti T, Francis S, Verstraelen H, and Jespers V. 2014. The vaginal microbiota: what have we learned after a decade of molecular characterization? PLoS One 9:e105998. 10.1371/journal.pone.0105998

Williams MR, and Gallo RL. 2015. The role of the skin microbiome in atopic dermatitis. Curr Allergy Asthma Rep 15:65. 10.1007/s11882-015-0567-4

Wolcott RD, Hanson JD, Rees EJ, Koenig LD, Phillips CD, Wolcott RA, Cox SB, and White JS. 2016. Analysis of the chronic wound microbiota of 2,963 patients by $16 \mathrm{~S}$ rDNA pyrosequencing. Wound Repair Regen 24:163-174. 10.1111/wrr.12370

World Health Organisation. 2016. Diabetes mellitus. Available at http://www.who.int/mediacentre/factsheets/fs138/en/2016).

Yosipovitch G, Tur E, Cohen O, and Rusecki Y. 1993. Skin surface pH in intertriginous areas in NIDDM patients. Possible correlation to candidal intertrigo. Diabetes Care 16:560-563.

Yu W, Yuan X, Xu X, Ding R, Pang L, Liu Y, Guo Y, Li H, Li M, Yuan J, Tang L, and Wen S. 2015. Reduced airway microbiota diversity is associated with elevated allergic respiratory inflammation. Ann Allergy Asthma Immunol 115:63-68. 10.1016/j.anai.2015.04.025

Zhang Y, and Zhang H. 2013. Microbiota associated with type 2 diabetes and its related complications. Food Science and Human Wellness 2:167-172. http://dx.doi.org/10.1016/j.fshw.2013.09.002 


\section{Figure 1 (on next page)}

Alpha diversity of skin and wounds

Box plots of 3 different alpha diversity measures, A) observed number of OTUs or richness, B) the Chao I estimator, and C) the Shannon index, based on OTUs clustered at $97 \%$ similarity for control skin, diabetic skin and diabetic wounds. Significant differences are indicated by asterix $*=p<0.05, * *=p<0.01 * * *=p<0.001$. 


\section{Figure 2 (on next page)}

Principal coordinates analysis of diabetic and control skin samples

Distances are based on the weighted unifrac metric, calculated using raw counts subjected to a variance stabilising transformation. 


\section{Figure 3 (on next page)}

The top 10 most abundant OTUs in diabetic and control skin per subject

The top 10 most abundant OTUs in A) control and B) diabetic skin per subject. Average abundances per person were calculated from data rarefied to 30000 sequences per sample. Genus assigned taxonomy is indicated in the legend, individual OTUs of the same genera are indicated with black lines. 


\section{Figure 4 (on next page)}

Boxplots of intra-individual differences over time in diabetic and non-diabetic skin microbial communities.

Inter-individual distances are also shown for comparison. The stability of non-diabetic skin

was higher (i.e. lower distances over time) than for diabetic skin, however this difference did not reach significance. (Kolmogorov-Smirnov test, $p=0.09$ ). 
Figure 5 (on next page)

The top 10 abundant OTUs in wounds per subject.

The top 10 abundant OTUs per subject in diabetic A) wound debridement and B) wound swab samples. Average abundances per group were calculated from data rarefied to 30000 sequences per sample. Genus assigned taxonomy is indicated in the legend, or family level where genus was unassigned. Individual OTUs of the same genera are indicated with black lines. 


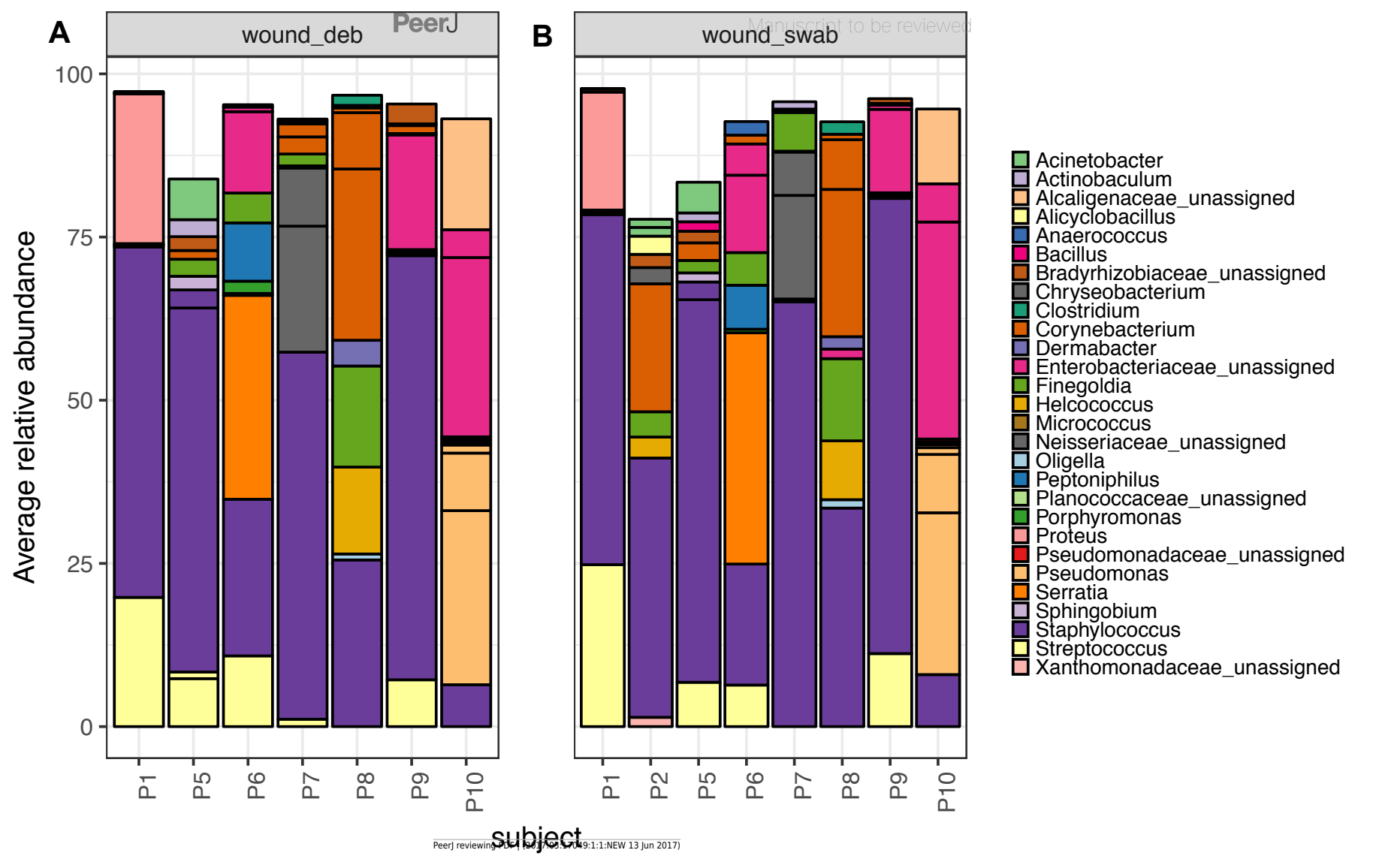


Figure $\mathbf{6}$ (on next page)

Relative abundance of the top 10 OTUs per patient over time

Patients 1-10 are represented individually in panels A-H. Wound area is overlaid as a red line and is represented as a percentage of the largest wound area measured over time. Relative abundances were calculated from data rarefied to 30000 sequences per sample. Genus assigned taxonomy is indicated in the legend, or family level where genus was unassigned. Individual OTUs of the same genera are indicated with black lines. 
A

$$
100
$$

100

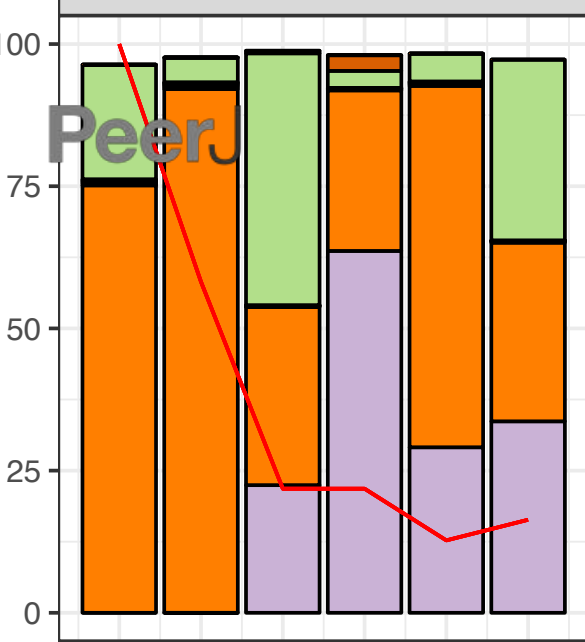

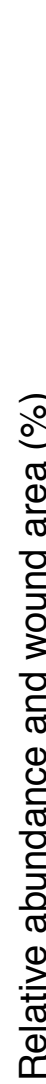

C
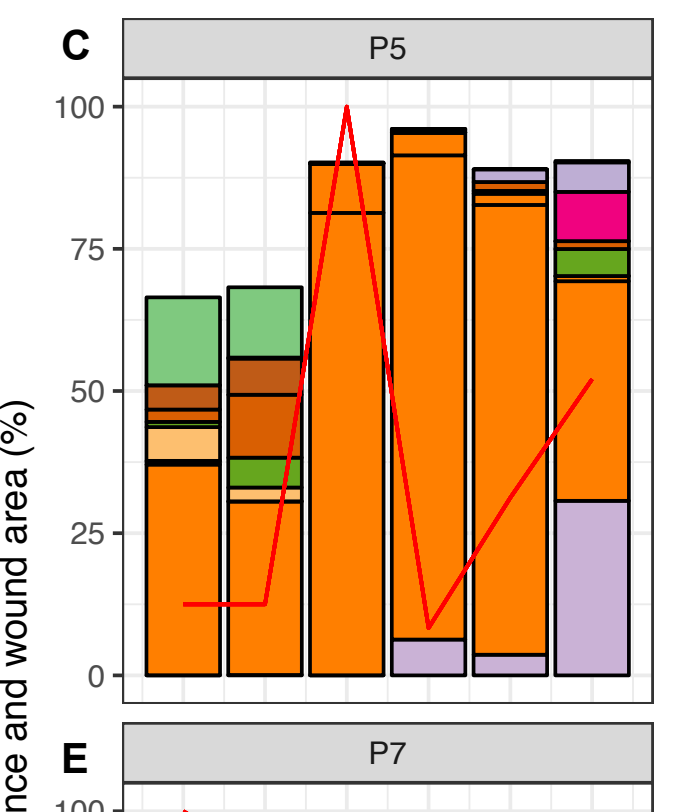

$100-$
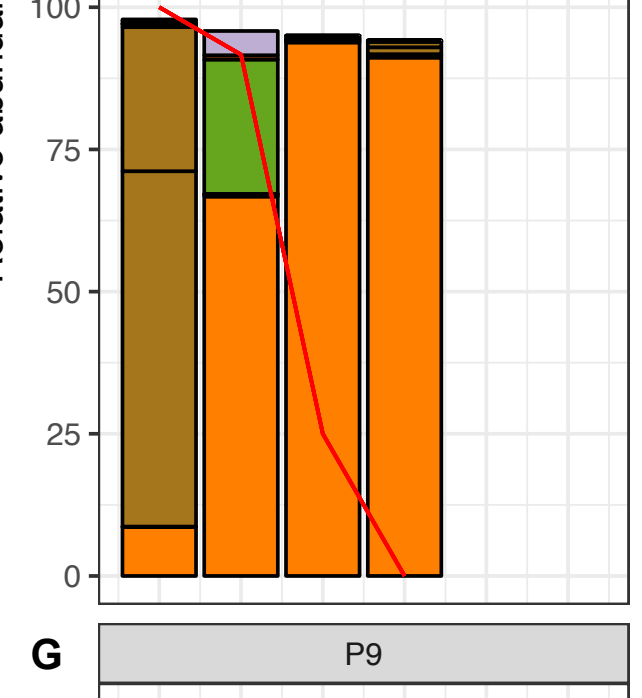$$
100
$$
.

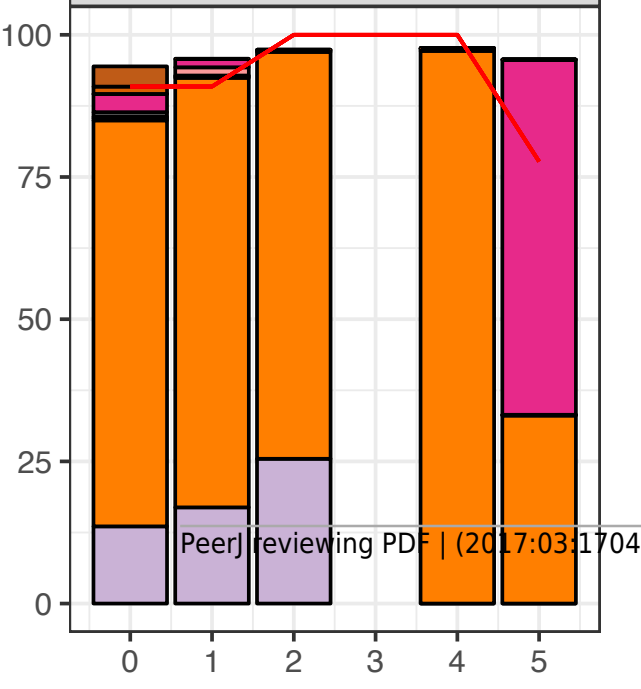

B

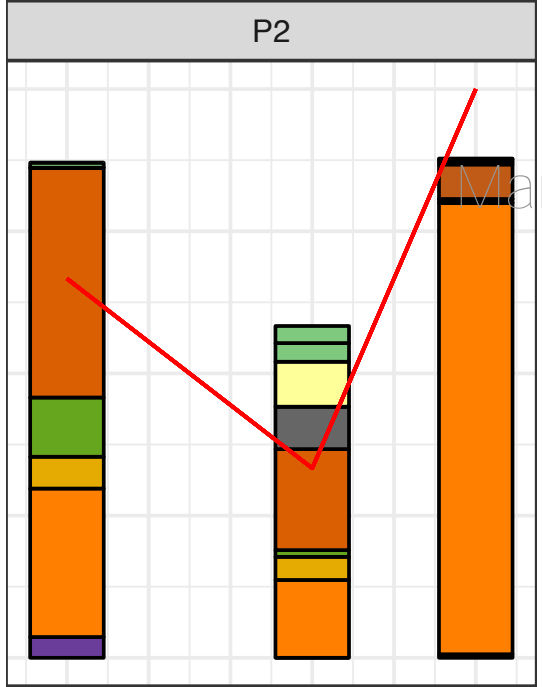

D

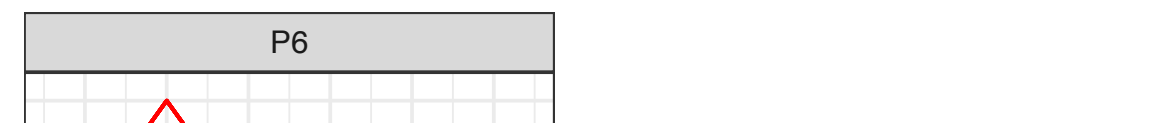

$\square$ Acinetobacter Actinobaculum

Alcaligenaceae_unassigned Alicyclobacillus Anaerococcus Bacillus

Bradyrhizobiaceae_unassigned Chryseobacterium Clostridium Corynebacterium Dermabacter Enterobacteriaceae_unassigned Finegoldia Helcococcus Neisseriaceae_unassigned Oligella

Peptoniphilus Porphyromonas

Proteus

Pseudomonadaceae_unassigned Pseudomonas

Serratia

Sphingobium

Staphylococcus

Streptococcus

Xanthomonadaceae_unassigned
H

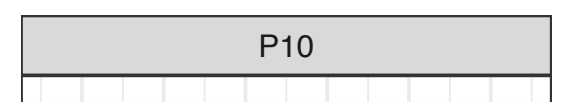

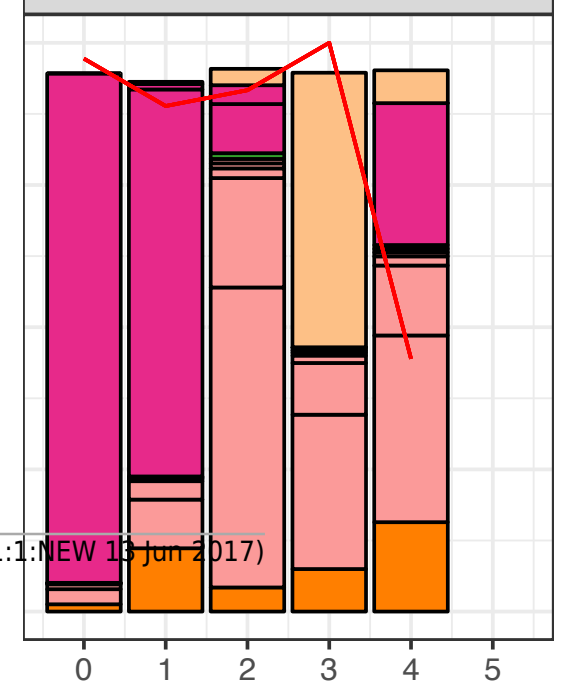




\section{Table $\mathbf{1}$ (on next page)}

Characteristics of diabetic and control cohorts.

Characteristics are shown for the diabetic and control subjects in the study. Average values with standard deviations are reported, including the range in brackets. 
1

2 Table 1: Characteristics of diabetic and control cohorts.

3

\begin{tabular}{|l|l|l|}
\hline & Diabetic & Control \\
\hline Age (years) & $68.9 \pm 8.2(58-81)$ & $62.8 \pm 13.4(50-81)$ \\
\hline BMI & $35.4 \pm 5.9(27.2-47.1)$ & $28.0 \pm 6.6(20.4-37.9)$ \\
\hline Males:Females & $5: 3$ & $2: 6$ \\
\hline
\end{tabular}

4

5 Characteristics are shown for the diabetic and control subjects in the study. Average values with standard deviations 6 are reported, including the range in brackets.

7 
Table 2 (on next page)

Primer sequences used in this study 


\section{Table 2: Primer sequences used in this study}

\begin{tabular}{|c|c|}
\hline Primer name & Sequence 5'-3' \\
\hline V4_forward_1 & AATGATACGGCGACCACCGAGATCTACACAACCAGTCTATGGTAATTGTGTGCCAGCMGCCGCGGTAA \\
\hline V4_forward_2 & AATGATACGGCGACCACCGAGATCTACACAACGCTAATATGGTAATTGTGTGCCAGCMGCCGCGGTAA \\
\hline V4_forward_3 & AATGATACGGCGACCACCGAGATCTACACAAGACTACTATGGTAATTGTGTGCCAGCMGCCGCGGTAA \\
\hline V4_forward_4 & AATGATACGGCGACCACCGAGATCTACACAATCGATATATGGTAATTGTGTGCCAGCMGCCGCGGTAA \\
\hline V4_forward_5 & AATGATACGGCGACCACCGAGATCTACACACCAATTGTATGGTAATTGTGTGCCAGCMGCCGCGGTAA \\
\hline V4_forward_6 & AATGATACGGCGACCACCGAGATCTACACACTGAAGTTATGGTAATTGTGTGCCAGCMGCCGCGGTAA \\
\hline V4_forward_7 & AATGATACGGCGACCACCGAGATCTACACATTGCCGCTATGGTAATTGTGTGCCAGCMGCCGCGGTAA \\
\hline V4_forward_8 & AATGATACGGCGACCACCGAGATCTACACCAACCTTATATGGTAATTGTGTGCCAGCMGCCGCGGTAA \\
\hline V4_forward_9 & AATGATACGGCGACCACCGAGATCTACACCCTAATAATATGGTAATTGTGTGCCAGCMGCCGCGGTAA \\
\hline V4_forward_10 & AATGATACGGCGACCACCGAGATCTACACCCTCTGATTATGGTAATTGTGTGCCAGCMGCCGCGGTAA \\
\hline V4_forward_14 & AATGATACGGCGACCACCGAGATCTACACGAACGGAGTATGGTAATTGTGTGCCAGCMGCCGCGGTAA \\
\hline V4_forward_16 & AATGATACGGCGACCACCGAGATCTACACGCGTTACCTATGGTAATTGTGTGCCAGCMGCCGCGGTAA \\
\hline V4_forward_18 & AATGATACGGCGACCACCGAGATCTACACGGATGCCATATGGTAATTGTGTGCCAGCMGCCGCGGTAA \\
\hline V4_forward_20 & AATGATACGGCGACCACCGAGATCTACACGTTGGCCGTATGGTAATTGTGTGCCAGCMGCCGCGGTAA \\
\hline V4_forward_22 & AATGATACGGCGACCACCGAGATCTACACTGACTGCTTATGGTAATTGTGTGCCAGCMGCCGCGGTAA \\
\hline V4_forward_24 & AATGATACGGCGACCACCGAGATCTACACTTCAGCGATATGGTAATTGTGTGCCAGCMGCCGCGGTAA \\
\hline V4_reverse_1 & CAAGCAGAAGACGGCATACGAGATAACCAGTCAGTCAGTCAGCCGGACTACHVGGGTWTCTAAT \\
\hline V4_reverse_7 & CAAGCAGAAGACGGCATACGAGATATTGCCGCAGTCAGTCAGCCGGACTACHVGGGTWTCTAAT \\
\hline V4_reverse_8 & CAAGCAGAAGACGGCATACGAGATCAACCTTAAGTCAGTCAGCCGGACTACHVGGGTWTCTAAT \\
\hline V4_reverse_9 & CAAGCAGAAGACGGCATACGAGATCCTAATAAAGTCAGTCAGCCGGACTACHVGGGTWTCTAAT \\
\hline
\end{tabular}




\begin{tabular}{|c|c|}
\hline V4_reverse_15 & CAAGCAGAAGACGGCATACGAGATGCCTACGCAGTCAGTCAGCCGGACTACHVGGGTWTCTAAT \\
\hline V4_reverse_16 & CAAGCAGAAGACGGCATACGAGATGCGTTACCAGTCAGTCAGCCGGACTACHVGGGTWTCTAAT \\
\hline V4_reverse_17 & CAAGCAGAAGACGGCATACGAGATGGAGGCTGAGTCAGTCAGCCGGACTACHVGGGTWTCTAAT \\
\hline V4_reverse_23 & CAAGCAGAAGACGGCATACGAGATTGGCGATTAGTCAGTCAGCCGGACTACHVGGGTWTCTAAT \\
\hline V4_reverse_24 & CAAGCAGAAGACGGCATACGAGATTTCAGCGAAGTCAGTCAGCCGGACTACHVGGGTWTCTAAT \\
\hline V4_reverse_25 & CAAGCAGAAGACGGCATACGAGATTTGGCTATAGTCAGTCAGCCGGACTACHVGGGTWTCTAAT \\
\hline Illumina_E_1 & AATGATACGGCGACCACCGA \\
\hline Illumina_E_2 & CAAGCAGAAGACGGCATACGA \\
\hline V4_read_1 & TATGGTAATTGTGTGCCAGCMGCCGCGGTAA \\
\hline V4_read_2 & AGTCAGTCAGCCGGACTACHVGGGTWTCTAAT \\
\hline V4_index_read & ATTAGAWACCCBDGTAGTCCGGCTGACTGACT \\
\hline
\end{tabular}

2 